\title{
Embedded acoustic long baseline localization system for autonomous underwater vehicles
}

\author{
Redouane Es-Sadaoui ${ }^{1}$, Jamal Khallaayoune ${ }^{2}$, Tamara Brizard ${ }^{3}$ \\ ${ }^{1,2}$ Department of Electronic, Microwave and Optic, National Institute of Posts and Telecommunications, Rabat, Morocco \\ ${ }^{3}$ Arkeocean SARL, Aspremont, France
}

\begin{abstract}
Article Info
Article history:

Received Apr 13, 2021

Revised Oct 11, 2021

Accepted Oct 18, 2021

\section{Keywords:}

Acoustic signal processing Autonomous underwater vehicles

Localization

Long baseline

Time-of-arrival

ABSTRACT

The attenuation of global positioning system (GPS) in water medium makes localization of autonomous uderwater vehicles (AUVs) particularly challenging. The long baseline (LBL) positioning system can extend GPS using beacons as references. This work aims at building an acoustic LBL-based system able to localize AUVs operating in swarms thanks to a small size acoustic transceiver embedded onboard AUVs and implementing range-based localization algorithms to estimate the swarm coordinates in real-time. The distances computation between navigating AUVs and fixed beacons were implemented in a digital signal processor (DSP) which computes the time-of-arrival (ToA) of incoming pure tone acoustic waves. The principle of design, hardware architecture, implementation, simulations and sea experiments are described in this paper. The experimental data showed an average deviation around $0.62 \mathrm{~m}$ when an AUV is placed at $45 \mathrm{~m}$ far away from a beacon. This deviation increases with distance: around $4.8 \mathrm{~m}$ measured at $500 \mathrm{~m}$. This performance can be improved by taking into consideration the two main factors examined in this paper, which are sound velocity profile and propagation model.
\end{abstract}

This is an open access article under the CC BY-SA license.

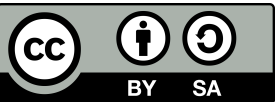

\section{Corresponding Author:}

Redouane Es-Sadaoui

Department of Electronic, Microwave and Optic

National Institute of Posts and Telecommunications

AV Allal El Fassi, Madinat Al Irfane, Rabat, Morocco

Email: essadaoui@inpt.ac.ma

\section{INTRODUCTION}

Nowadays, navies are interested in deploying autonomous uderwater vehicles (AUV) swarms to help survey, classify and map the largest unexplored surface on the earth with high accuracy and at a fast timing; see [1]-[3]. However, gathered data can only be processed meaningfully when referenced to the coordinate of the sensors, giving localization a critical role. Various localization algorithms have been studied in terrestrial sensor networks [4], [5], but they cannot be applied directly to AUVs because of attenuation of global positioning system (GPS) and radio frequency (RF) signals underwater, therefore acoustic waves are needed to achieve long-range sensing or communication [6], [7].

Typically, localization methods described in the literature are dependent to underwater sensor networks (UWASNs) and very varied both in terms of the number of transmitters and receivers used and their geometric configuration; see [4], [8]. Most of the listed technologies are individually interesting but they must be judiciously combined to give an easy-to-use system adapted to the application specifications. They must also be deploy-able and can be used reliably and efficiently under the conditions in which AUVs operate. Long 
baseline (LBL) is one of the most common "range-based" systems [9]-[11] that performs localization of AUVs in two stages: We first measure parameters which can be distance, this information is then used in a second calculation step to estimate the position of the AUV.

This paper addresses the two main challenges raised by the implementation of LBL-based localization for one or multiple AUVs underwater: i) The "Underwater localization challenge" where each AUV should be able to detect and measure the relative distance to anchor nodes and computes its absolute universal transverse mercator (UTM) coordinate; ii) The "Accurate ranging challenge" characterizing the acoustic distance performance by investigating the main factors that affect the propagation of sound waves in submarine environment.

\section{REVIEW OF AUV LOCALIZATION SYSTEMS}

\subsection{Recent AUV swarm projects}

This section shows some example of interesting AUV swarms projects appeared in the last five years. The COMET project, labeled by the Pôle Mer Bretagne Atlantique, offers an interesting approach for the coordination of a group of AUVs where one of the vehicles, the "swarm leader", has a high-performance inertial unit that he recalls on a precise position either by acoustic communication with a boat, or during occasional returns to the surface. The "swarm leader" then communicates this positioning information to the other AUVs, which in turn reset. This allows the swarm to have precise positioning without having to equip each robot with a heavy and expensive inertial unit. Nevertheless, this system is very centralized and shows its operational limits in the event of failure of the "leader of the swarm". Redefining the function of the "swarm leader" within AUVs could appear attractive at first glance, but it will significantly increase costs by the proliferation of expensive inertial units and will complicate the operational management of AUVs when a new "swarm leader" will have to replace its failing predecessor. Figure 1 (a) shows an example of AUV used by the COMET project [2], which allows acoustic imagery for offshore operations. Thanks to its acoustic communication system (RTSYS core expertise) and long baseline (sparse-LBL) positioning algorithms, COMET is able to navigate up to 20 hours with most accurate position of the market. It can also host various payloads and proceed to chemical-physical assessment. This project was concluded in June 2016 with successful sea trials.

Manley and Smith [1] started the design and development effort of their first micro-UUV in mid2015, a new, highly flexible, open source autonomous undersea vehicle that provides a state-of-the-art, low cost solution ideally suited for developers of autonomy and behaviors, power systems, subsea sensors, and other new payloads. In 2018, Riptide's initiative at the Advanced Naval Technology Exercise to deploy 6 small AUVs (image shown in Figure 1 (b) ) in a coordinated mission is also noteworthy because it shows the interest aroused by small size for new military missions. The unit cost of this potentially reasonable new generation of AUVs makes it financially possible to deploy very large packs. The micro AUV is equipped with depth and temperature measuring sensors. It is also fitted with ultra-compact $600 \mathrm{kHz}$ pathfinder doppler velocity log (DVL) supplied by teledyne RD instruments (TRDI).

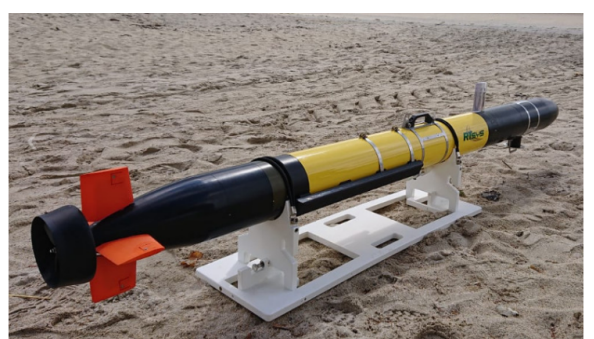

(a)

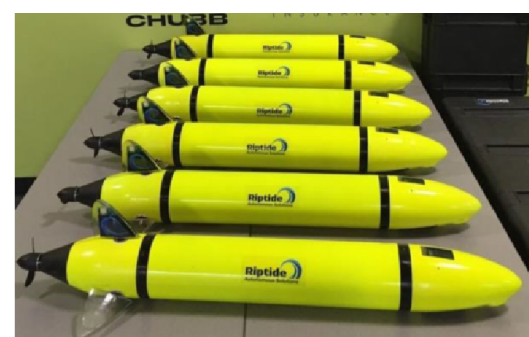

(b)

Figure 1. Picture of: (a) COMET AUV, 300 meter depth [2] and (b) 6 riptide AUVs [1]

\subsection{LBL localization scheme}

The example drawn in Figure 2 (a), considers the acoustic LBL with transponders : An interrogator (A) is installed on the AUV and several acoustic transponders ( 3 minimum) are immersed at fixed positions on the bottom. The interrogator transmits an acoustic signal at time T0 which is received by the transponders (B), (C) and (D) which in turn emit, after a known response time DR, personalized and specific to each, an acoustic 
signal response that the AUV intercepts at times TB, TC and TD. The AUV thus calculates the distance to each of the transponders by subtracting from the time of flight of the acoustic signals the response time DR specific to each, by dividing by 2 to hold the round trip, and by multiplying by the speed of sound in water.

However, for a swarm, as illustrated in Figure 2 (b), the reality is not that simple, there will be a large number of AUVs wanting to access the same resource that transponders constitute. It will be necessary to set up a time-sharing system in periods during which a single AUV can interrogate and receive the responses from the transponders. Time division multiple access (TDMA) as described in [12] is an example of time sharing.

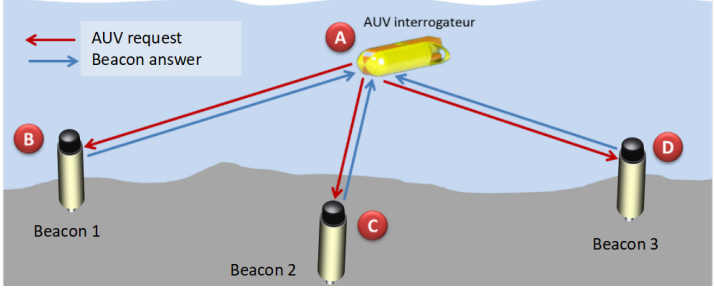

(a)

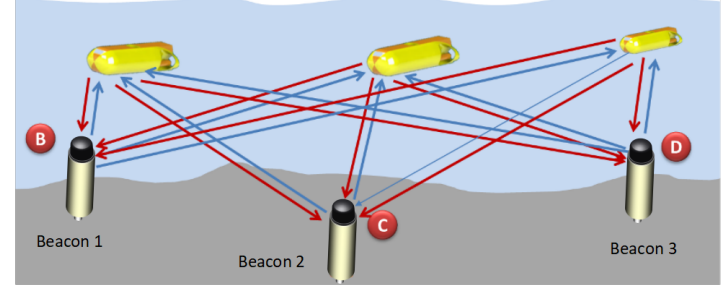

(b)

Figure 2. Diagram showing an example of LBL system with: (a) one AUVs and (b) multiple AUVs

\subsection{Factors affecting accuracy}

\subsubsection{Sound velocity profile}

Sound travels faster with increasing temperature, salinity, and pressure [13]. Thus, speed measurements will have to be carried out on site with sound velocity profile (SVP) probes. A simple empirical equation for the speed of sound in sea water, with reasonable accuracy for the world's oceans, is due to Mackenzie [14]. We selected this model for computing the sound speed because its standard error is small, the formula contains fewer items and its depth range can reach to $8000 \mathrm{~m}$.

$$
c(T, S, D)=A_{1}+A_{2} \cdot T+A_{3} \cdot T^{2}+A_{4} \cdot T^{3}+A_{5}(S-35)+A_{6} \cdot D+A_{7} \cdot D^{2}+A_{8} \cdot T \cdot(S-35)+A_{9} \cdot T \cdot D^{3}
$$

where $T$ is the temperature in degrees Celsius. $S$ is the Salinity in parts per thousand. $D$ is the depth in meters. The constants used are:

$$
\begin{aligned}
& A 1=1448.96, A 2=4.591, A 3=-5.304 .10^{-} 2, A 4=2.374 .10^{-} 4, A 5=1.340 \\
& A 6=1.63 .10^{-} 2, A 7=1.675 .10^{-} 7, A 8=-1.025 .10^{-} 2, A 9=-7.139 .10^{-} 13
\end{aligned}
$$

\subsubsection{Propagation model}

Converting measured time-of-flight (ToF) to radial distance requires taking propagation into account and using an appropriate propagation model [15]-[17]. The SONAR equation [13] will be used to compute the received signal-to-noise ratio (SNR) level from the transmitted signal level, taking into account transmission loss and noise level and the effect of the SNR level on the measurement accuracy should be investigated. The system equation of a passive sonar system is written:

$$
S N R=S L-T L-N L+D I+10 \log T-P L>D T
$$

where $S N R$ is the signal to noise ratio of acoustic pulse arrived at the the receiver signal processing Unit. $S L$ is the sound level of the transmitter. $T L$ is the transmission loss, it measures the attenuation of the signal in $\mathrm{dB}$ between the transmitter (at $1 \mathrm{~m}$ therefore) and the receiver (in water before application of the reception antenna treatment). $N L$ is the noise at reception. $D I$ is the antenna gain. $D T$ is the detection threshold. $10 \log T$ is a gain which comes from the signal processing.

The Transmission Loss is generally calculated with an adequate propagation program. In the simplified case of an isosceles propagation a distinction is made between a loss "in $1 / R^{2}$ " (which is written $-20 \log R$ in $\mathrm{dB}$ with $\mathrm{R}$ in meter) and a loss by absorption which is proportional to the distance traveled. By taking units of distance in Kilometers we finally get:

$$
T L=60+20 \log R+\alpha \cdot R
$$


The $60 \mathrm{~dB}$ comes from the fact that emission levels are measured at $1 \mathrm{~m}$. The term $T L$ is measured in $\mathrm{dB} / \mathrm{Km}$ and is a function of the frequency (but also of salinity, $\mathrm{PH}$ and immersion). In addition, the absorption of sound in seawater should be considered: The absorption profile published in 1982 by Francois and Garrison [18], drew together a wide range of previous measurements including the earlier publication of Fisher and Simmons in 1977 [19].

\section{PROPOSED SOLUTION}

\subsection{Hardware architecture}

The proposed solution combines sophisticated building blocks based on a low volume, low weight and low cost acoustic device able to transmit and receive acoustic pulses. The electronic board was designed to be packaged in two versions: i) Onboard the AUV : shown in Figure 3 (a), to detect pulses from beacons and provide the AUV with the acoustic distance and absolute position; ii) onboard the beacons: shown in Figure 3 (b), to run in pre-programmed modes and transmit acoustic pulses autonomously.

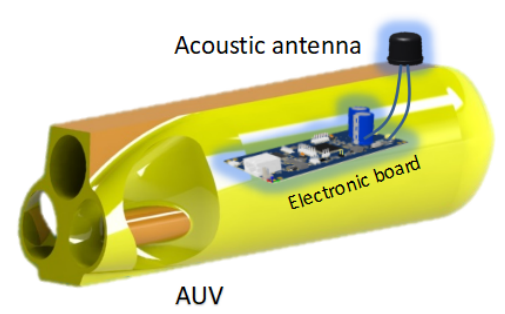

(a)

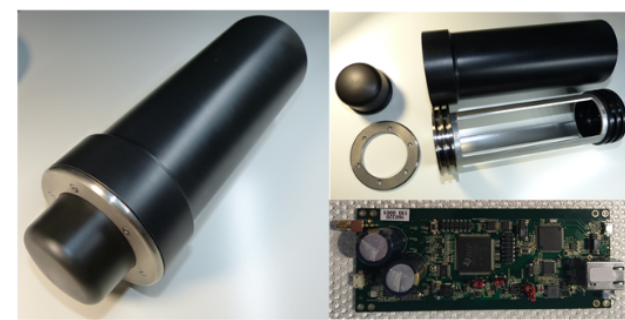

(b)

Figure 3. Electronic board packaging, onboard: (a) the AUV and (b) the standalone beacon

\subsection{Position estimation method}

Figure 4 shows the system components. Three acoustic beacons are deployed on the seabed and covering a survey zone of $1 \times 1 \mathrm{~km}$. The beacons are synchronized and transmit acoustic pulses at programmed time of emission (ToE). The GPS positions of three beacons (UTM coordinates [x1,y1],[x2,y2] and [x3,y3]) are known.

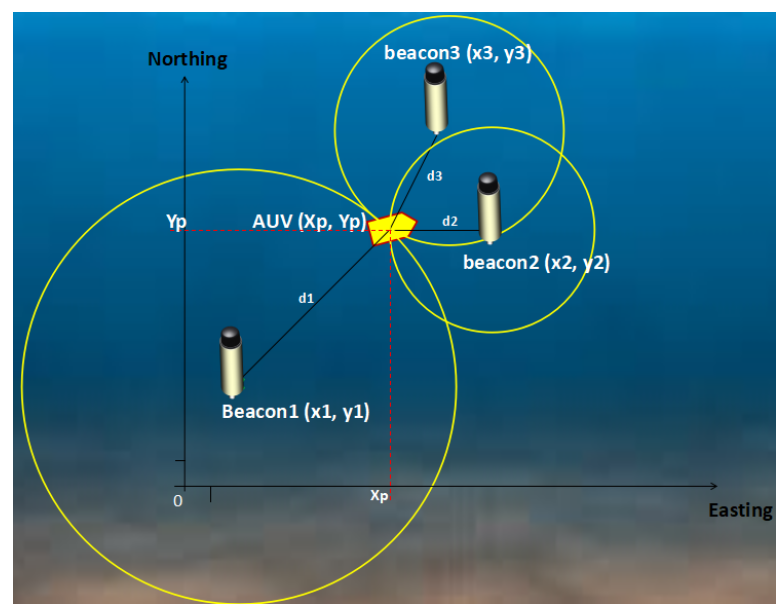

Figure 4. Diagram showing the LBL localization system components in 2D space. The position of the AUV [xp,yp] can be calculated using trilateration [20]

AUVs will be first synchronized with the same time reference of the beacons and then deployed in the survey zone. Thanks to the acoustic transceiver onboard the AUV, they will detect acoustic pulses from the beacons and recognize them by matching the ToAs to the programmed ToEs. Knowing the sound velocity 
of water, the distance (d) to the beacon is estimated (5) by calculating the time of flight (ToF) which is the difference between the ToA and the ToE $(T o A-T o E)$.

$$
d=T o F * \text { Svelocity }
$$

Once the distances between AUV and at least tree beacons are known, the absolute position of the AUV can be calculated using trilateration [20], which consists in using relative distances to anchor nodes or beacons to estimate the location of the AUV searched. With three beacons, the 2-D coordinate (xp, yp) is determined by solving the equations of three circles (6), for $n=1,2,3$.

$$
(x-x p)^{2}+(y-y p)^{2}=d^{2}
$$

\subsection{Acoustic chain}

The acoustic chain blocks are illustrated in Figure 5. Received signals pass through amplification and filtering, then sampled by the ADC converter [21]. An energy detector mechanism was implemented on the digital signal processor (DSP) [22] to detect and measure the time of arrival of incoming pulses. The acoustic chain processing was covered in this paper [23].

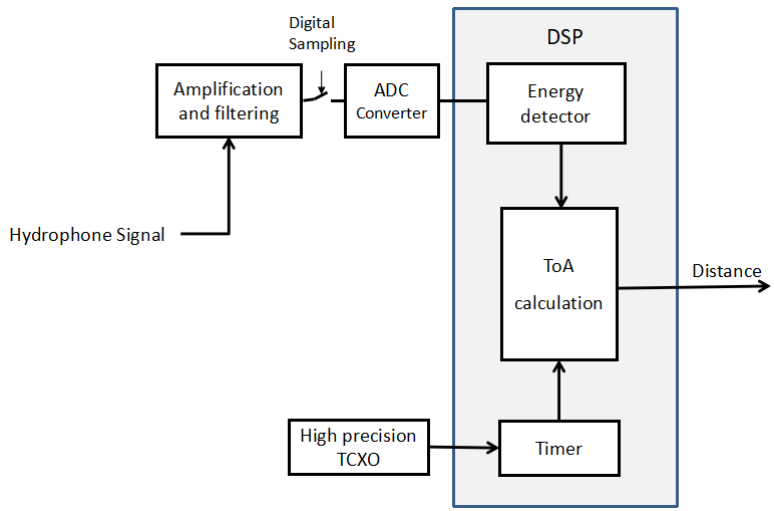

Figure 5. The acoustic chain blocks

\subsection{Simulation}

MATLAB simulations have been conducted in order to qualify the performance of the acoustic chain in detecting pure tone sine waves sent by the acoustic pinger and measuring accurately the ToA of incoming pulses. The example shown in Figure 6 is for a signal of SNR around $66 \mathrm{~dB}$. In blue is the acoustic pulse (shaped in blackman window of $12 \mathrm{~ms}$ duration). In Red is the detection status which goes high when the difference between the pulse energy and the sea noise level exceeds a detection threshold $12 \mathrm{~dB}$.

The measured detection ToAs values depending on the SNR level are summarized in Table 1. The obtained results confirm that the detection ToA measurements are smaller with high SNR and increase when the SNR of inputs signal decreases(up to $8.92 \mathrm{~ms}$ at $\mathrm{SNR}=12 \mathrm{~dB}$ )

Table 1. Simulated ToA deviation depending on the SNR level

\begin{tabular}{ccc}
\hline Contrast level (decibel) & ToA deviation (millisecond) & range deviation (meter) \\
\hline $66 \mathrm{~dB}$ & $2.35 \mathrm{~ms}$ & $3.53 \mathrm{~m}$ \\
$46 \mathrm{~dB}$ & $3.58 \mathrm{~ms}$ & $5.37 \mathrm{~m}$ \\
$26 \mathrm{~dB}$ & $5.63 \mathrm{~ms}$ & $8.44 \mathrm{~m}$ \\
$16 \mathrm{~dB}$ & $7.35 \mathrm{~ms}$ & $11.03 \mathrm{~m}$ \\
$12 \mathrm{~dB}$ & $8.92 \mathrm{~ms}$ & $13.38 \mathrm{~m}$ \\
\hline
\end{tabular}




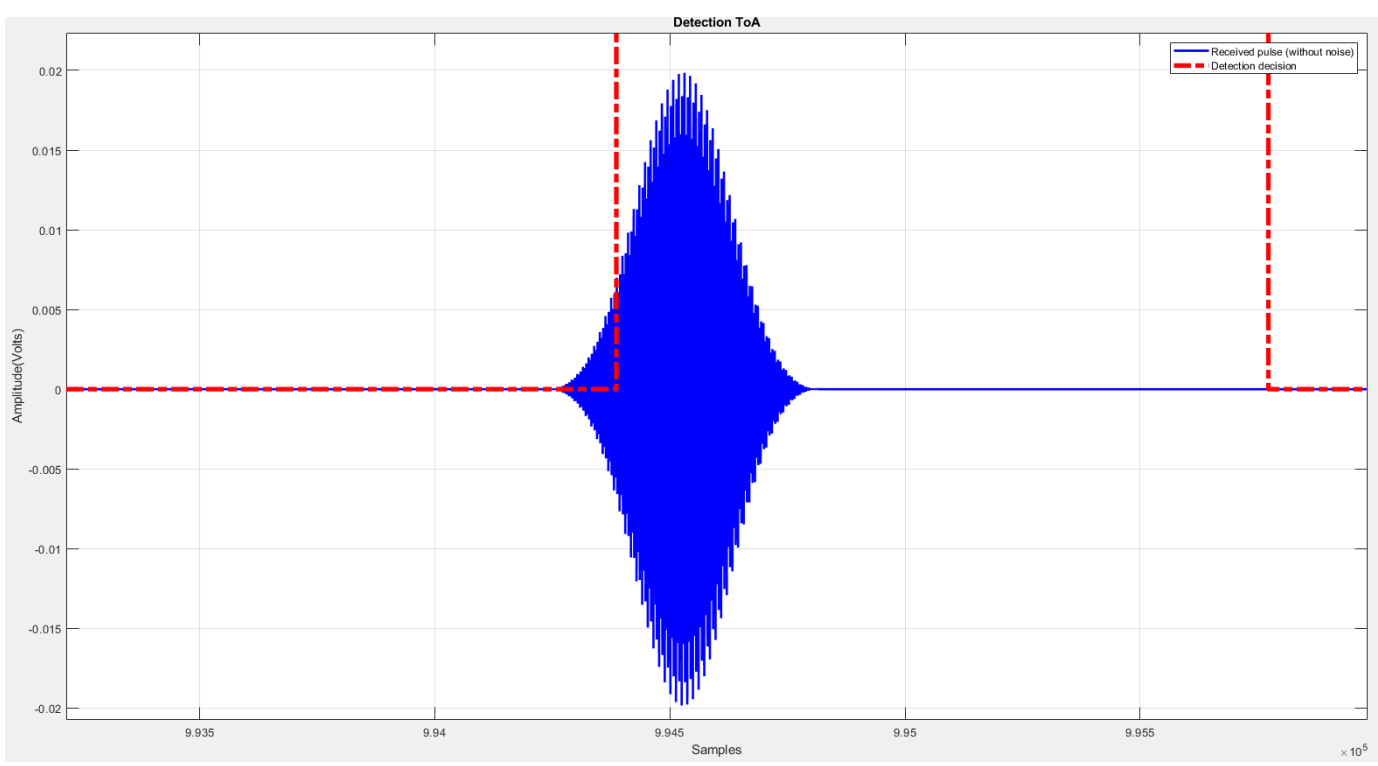

Figure 6. Measured detection ToA in MATLAB: Input signal is a blackman pulse, frequency $=20 \mathrm{KHz}$, duration $=12 \mathrm{~ms}, \mathrm{SNR}=66 \mathrm{~dB}$; Horizontal axis is Time in samples; Vertical axis is Amplitude in Volts

\section{SEA EXPERIMENTS RESULTS}

\subsection{Experimental platform}

The developed system was tested in Guerlédan Lake, France. This site provides an easy to access platform that allow us to qualify the performance of our system within $1 \mathrm{~km}$ line of sight range and 30 meters water depth. Photos of the experimental platform is shown in Figure 7. The AUV was deployed in line of sight with the acoustic beacon. The Sound velocity was measured around $1480 \mathrm{~m} / \mathrm{s}$ using the monitor SVP from Valeport [24]. The acoustic beacon software was set to send pulses periodically (every second) at $20 \mathrm{kHz}$ frequency and $12 \mathrm{~ms}$ duration. The acoustic transceiver software was set to listen for acoustic pulses at $20 \mathrm{kHz}$ frequency channel and measure the ToAs of detected signals. The measured data is logged into a flash memory.

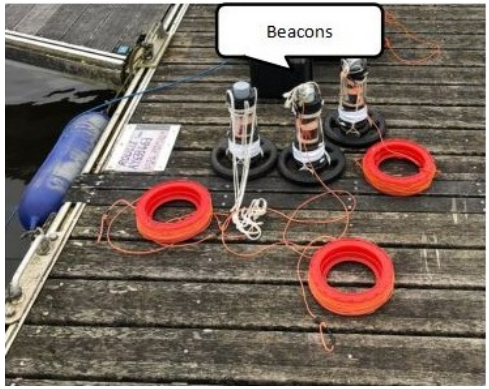

(a)

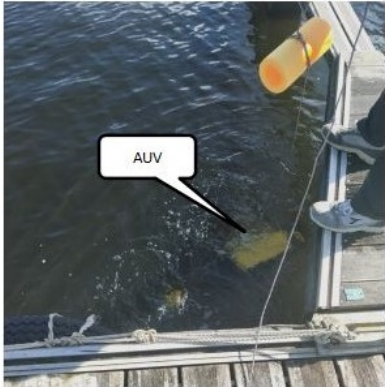

(b)

Figure 7. Photos of the experimental platform: (a) acoustic beacons on the deck and (b) AUV deployed in water

\subsection{Results and discussion}

Experimental data were obtained by varying the distance between a time-synchronized acoustic beacon and AUV and measuring the ToAs of incoming pulses at different locations: Beacon at $15 \mathrm{~m}$, beacon at 45 $\mathrm{m}$ and beacon at $500 \mathrm{~m}$. The recorded ToAs are logged into the flash memory in reference to the appropriate timestamp. Figure 8 gives a comparison between the deviations measured at 15,45 and $500 \mathrm{~m}$. We notice that the error appears as an offset value which is function the distance: The deviation is smaller at near field (15 $\mathrm{m})$ 
and become bigger when we moved the beacon to $500 \mathrm{~m}$ far away from the AUV. Table 2 summarizes the measured deviations: minimum and maximum deviation are respectively the minimum and maximum difference between the measured and true distance. The average deviation is the arithmetical mean of the given deviations while the peak to peak variance is simply calculated using the absolute difference between the maximum and the minimum deviation.

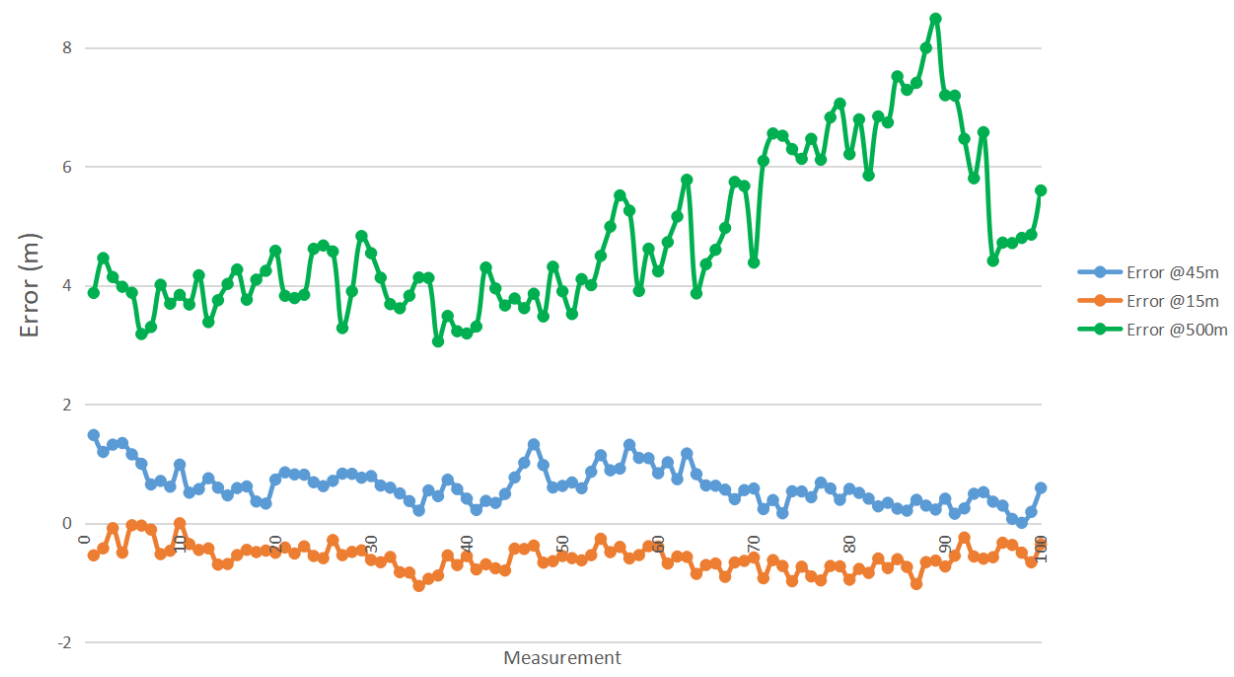

Figure 8. Distance measurement deviation: The bottom line is the deviation at $15 \mathrm{~m}$; The middle line is the deviation at $45 \mathrm{~m}$; The top line is the deviation at $500 \mathrm{~m}$; The horizontal axis is measurement record; and The vertical axis is deviation in meters

Table 2. Measured deviations: minimum, maximum, average and variance at 15, 45 and $500 \mathrm{~m}$

\begin{tabular}{cccc}
\hline Parameter & At $15 \mathrm{~m}$ & At $45 \mathrm{~m}$ & At $500 \mathrm{~m}$ \\
\hline Max deviation $(\mathrm{m})$ & $-0.0045 \mathrm{~m}$ & $1.4805 \mathrm{~m}$ & $8.485 \mathrm{~m}$ \\
Min deviation $(\mathrm{m})$ & $-1.059 \mathrm{~m}$ & $0.003 \mathrm{~m}$ & $3.0535 \mathrm{~m}$ \\
Average deviation $(\mathrm{m})$ & $-0.58478 \mathrm{~m}$ & $0.62625 \mathrm{~m}$ & $4.8000 \mathrm{~m}$ \\
Variance $(\mathrm{m})$ & $1.0545 \mathrm{~m}$ & $1.4775 \mathrm{~m}$ & $5.4315 \mathrm{~m}$ \\
\hline
\end{tabular}

\subsubsection{Distance accuracy depending on the SNR}

In this test, we fixed the distance between the beacon and the AUV at 45 meters and we managed to variate the SNR level of pulses received by AUV using a remote operating vehicle (ROV) [25] placed close to the AUV but not in the trajectory of incoming pulses (Figure 9 (a)). We can considerably notice that the SNR level decreases when the ROV activates their motors. This variation in SNR level directly affects the acoustic distance measured by the AUV as explained in Section 3.4. Figure 9 (b) shows the obtained distance measurement depending on the SNR level variation at fixed location (45 meters). The profile simulated in Table 1 can be applied here to compensate the static deviation (offset) depending on the variation of SNR level.

\subsubsection{SNR attenuation depending on the distance}

The raw value of SNR level (in $\mathrm{dB}$ ) was measured by the DSP based on the contrast between the maximum value of the received pulse energy $(\max R x E n)$ and the sea noise level (NoiseEn) as shown in (7):

$$
S N R=20 . \log \frac{\max R x E n}{\text { NoiseEn }}
$$

these values were measured: around $35 \mathrm{~dB}$ at $15 \mathrm{~m}, 33 \mathrm{~dB}$ at $45 \mathrm{~m}, 28 \mathrm{~dB}$ at $320 \mathrm{~m}$ and $25 \mathrm{~dB}$ at $500 \mathrm{~m}$. Values at each location were averaged using the arithmetical mean of raw values. 


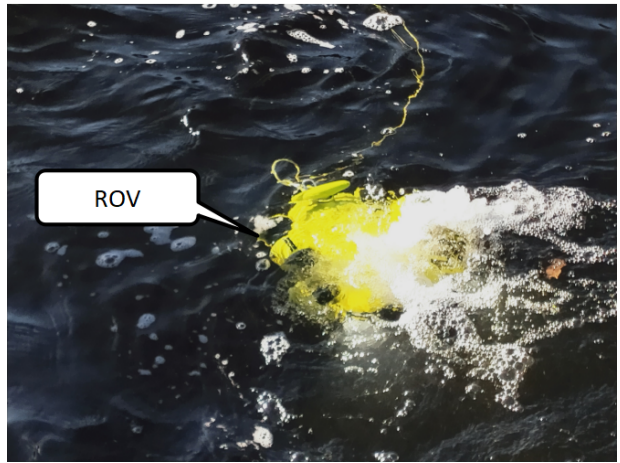

(a)

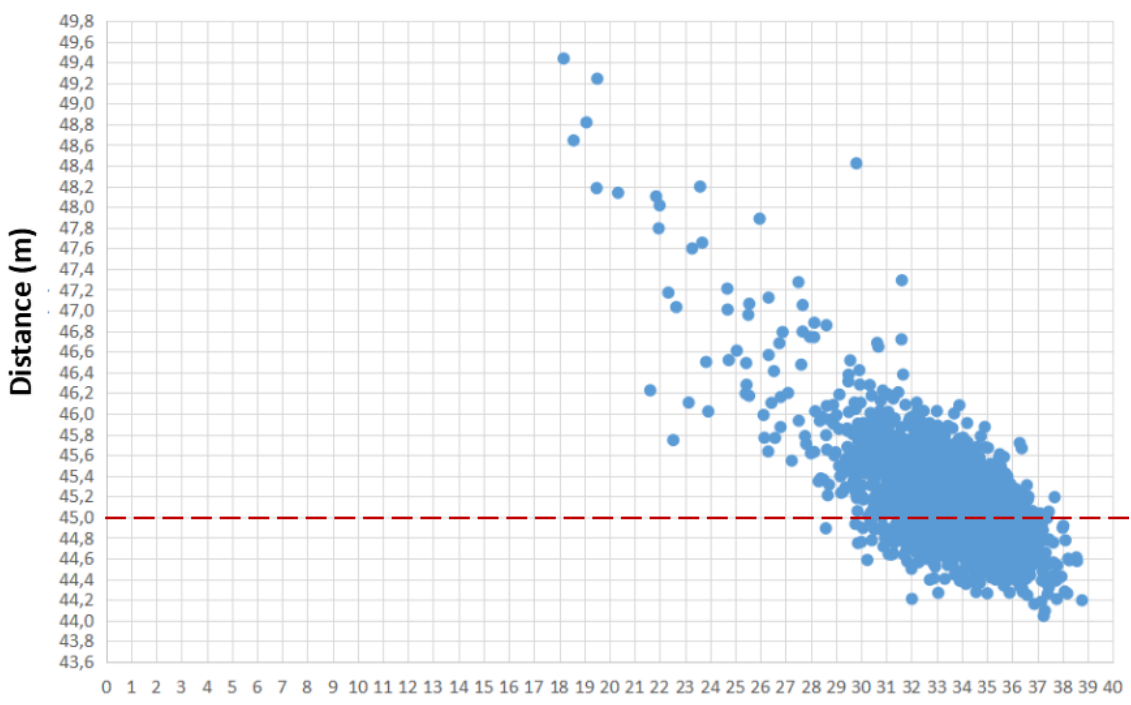

SNR (dB)

(b)

Figure 9. Picture of: (a) Fifish ROV [25] used to pollute the environment and (b) Distance accuracy profile measured depending on the SNR level variation, The true value is $45 \mathrm{~m}$

\section{CONCLUSION}

The ultimate objective of this work is to develop an acoustic system that allows AUVs operating in swarm to localize themselves relative to fixed beacons with known GPS positions. This paper presented the initial results obtained with an LBL-based localization system and outlined the main factors affecting its performance. A particular challenge associated with the position estimation is in characterizing the accuracy of acoustic distance measured by the AUV relative to each beacon, which depends on the sound propagation properties in the water environment. The ToA deviation depending on the SNR was characterized in MATLAB simulation. Sea experiments were performed using a fixed beacon deployed on the seafloor which sends pure tone acoustic pulses continuously. The acoustic transceiver onboard the AUV detects incoming pulses and measures the ToAs. The acoustic distance between beacon and AUV was evaluated at different locations: An offset of around $0.58 \mathrm{~m}$ average deviation was obtained at 15 meters distance and $4.8 \mathrm{~m}$ at 500 meters. This offset can be compensated by taking into consideration the SNR level profile depending on the distance variation. As perspective, the efficiency of this model should be investigated with large number of AUV swarms.

\section{ACKNOWLEDGMENT}

This work was supported by Arkeocean SARL, France. 


\section{REFERENCES}

[1] J. Manley and J. Smith, "Taking uuvs faster, further and deeper," Marine Technology Reporter Magazine, pp. 42-46, 2018. [Online]. Available: https://magazines.marinelink.com/Magazines/MarineTechnology/201806/content/takingfurther-deeper-538551

[2] COMET-300, "Technical manual: COMET-300 MAN PORTABLE AUTONOMOUS UNDERWATER VEHICLE," RTSYS, Rev. 003 ed., 2021.

[3] J. Lu, X. Chen, M. Luo, and Y. Zhou, "Cooperative localization for multiple auvs based on the rough estimation of the measurements," Applied Soft Computing, vol. 91, 2020, doi: 10.1016/j.asoc.2020.106197.

[4] I. F. Akyildiz, D. Pompili, and T. Melodia, "Underwater acoustic sensor networks: research challenges," Ad Hoc Networks, vol. 3, no. 3, pp. 257-279, 2005, doi: 10.1016/j.adhoc.2005.01.004.

[5] J. Rezazadeh, M. Moradi, and A. S. Ismail, "Fundamental metrics for wireless sensor networks localization," International Journal of Electrical and Computer Engineering (IJECE), vol. 2, no. 4, pp. 452-455, August 2012, doi: 10.11591/ijece.v2i4.247.

[6] N. Chapman, "Chapter 9 - inverse methods in underwater acoustics," in Applied Underwater Acoustics, T. H. Neighbors and D. Bradley, Eds. Elsevier, 2017, pp. 553-585, doi: 10.1016/B978-0-12-811240-3.00009-6.

[7] D. B. Kilfoyle and A. B. Baggeroer, "The state of the art in underwater acoustic telemetry," IEEE Journal of Oceanic Engineering, vol. 25, no. 1, pp. 4-27, Jan. 2000, doi: 10.1109/48.820733.

[8] L. Bjorno, "Chapter 14 - underwater acoustic measurements and their applications," in Applied Underwater Acoustics, T. H. Neighbors and D. Bradley, Eds. Elsevier, 2017, pp. 889-947, doi: 10.1016/B978-0-12-811240-3.00014-X.

[9] N. Kussat, C. Chadwell, and R. Zimmerman, "Absolute positioning of an autonomous underwater vehicle using gps and acoustic measurements," in IEEE Journal of Oceanic Engineering, vol. 30, no. 1, pp. 153-164, Jan. 2005, doi: 10.1109/JOE.2004.835249.

[10] N. Crasta, D. Moreno-Salinas, A. Pascoal, and J. Aranda, "Range-based cooperative underwater target localization," IFAC-PapersOnLine, vol. 50, no. 1, pp. 12366-12373, 2017, doi: 10.1016/j.ifacol.2017.08.2069.

[11] X. Wei, X. Wang, X. Bai, S. Bai, and J. Liu, "Autonomous underwater vehicles localisation in mobile underwater networks,” Int. J. Sens. Networks, vol. 23, no. 1, pp. 61-71, 2017, doi: 10.1504/IJSNET.2017.080664.

[12] P. Mandal, "Performance comparison of tdma variants for underwater acoustic sensor networks," Journal of Network and Computer Applications, vol. 92, pp. 20-30, 2017, doi: 10.1016/j.jnca.2017.01.030.

[13] R. P. Hodges, Underwater acoustics : Analysis, Design and Performance of Sonar, first edition ed. Hoboken, NJ: John Wiley and sons, 2010.

[14] K. V. Mackenzie, "Nine-term equation for sound speed in the oceans," The Journal of the Acoustical Society of America, vol. 70, no. 3, pp. 807-812, 1981, doi: 10.1121/1.386920.

[15] W. Kuperman and P. Roux, "Underwater Acoustics," Springer Handbooks, In: Rossing T. (eds) Springer Handbook of Acoustics, Springer, New York, 2007, pp. 149-204, doi: 10.1007/978-0-387-30425-0_5.

[16] M. Stojanovic and J. Preisig, "Underwater acoustic communication channels: Propagation models and statistical characterization,” IEEE Communications Magazine, vol. 47, no. 1, pp. 84-89, 2009, doi: 10.1109/MCOM.2009.4752682.

[17] P. A. van Walree, "Propagation and scattering effects in underwater acoustic communication channels," IEEE Journal of Oceanic Engineering, vol. 38, no. 4, pp. 614-631, Oct. 2013, doi: 10.1109/JOE.2013.2278913.

[18] R. E. Francois and G. R. Garrison, "Sound absorption based on ocean measurements: Part I: Pure water and magnesium sulfate contributions," Journal of the Acoustical Society of America, vol. 72, no. 3, pp. 896-907, 1982, doi: 10.1121/1.388170.

[19] F. H. Fisher and V. P. Simmons, "Sound absorption in sea water," Journal of the Acoustical Society of America, vol. 62 , no. 3, pp. 558-564, 1977, doi: 10.1121/1.381574.

[20] A. Toky, R. P. Singh, and S. Das, "Localization schemes for underwater acoustic sensor networks - a review," Computer Science Review, vol. 37, 2020, doi: 10.1016/j.cosrev.2020.100241.

[21] Cirrus Logic, Technical manual : CS5343/4 $98 \mathrm{~dB}, 96 \mathrm{kHz}$, Low Power Stereo A/D Converters, doc. number ADCC5344, 2015. [Online]. Available: https://www.cirrus.com/products/cs5343-44/

[22] Technical manual :TMS320C54x, TMS320LC54x, TMS320VC54x FIXED-POINT DIGITAL SIGNAL PROCESSORS, Texas Instruments, doc. number SPRS039C, 1999.

[23] R. Es-Sadaoui, J. Khallaayoune, and T. Brizard, "Design and performance measurement of energy-based acoustic signal detection with autonomous underwater vehicles," International Journal of Advanced Computer Science and Applications, vol. 12, no. 4, 2021, doi: 10.14569/IJACSA.2021.0120490.

[24] Valeport, Datasheet Reference: MONITOR SVP 500, October 2011, version 2B.

[25] Fifish v6/v6s ROV Quick Start Guide, QySea, version 1.4, 2021. [Online]. Available: https://www.qysea.com/uploads/file/FIFISH_V6_Quick_Start_V1.4_EN.pdf 